\title{
THE EFFECT OF ENDOSCOPIC INJECTIONS OF DEXTRANOMER BASED IMPLANTS ON CONTINENCE AND BLADDER CAPACITY: A PROSPECTIVE STUDY OF 31 PATIENTS
}

\author{
H. B. LOTTMANN, M. MARGARYAN, M. BERNUY, M.-J. ROUFFET, M.-O. BAU, A. EL-GHONEIMI, \\ Y. AIGRAIN, A. STENBERG* AND G. LÄCKGREN* \\ From the Pediatric Urology Department, Fondation Hopital Saint-Joseph, Paris, the Urotherapy Unit, Fondation Ellen Poidatz, \\ St. Fargeau Ponthierry, the Pediatric Surgery Department, Hopital Robert Debré, Paris, France, and the Section of Urology, \\ University Children's Hospital, Uppsala, Sweden
}

\section{ABSTRACT}

Purpose: A prospective study was conducted to assess the efficacy of dextranomer based implants as a new bulking agent for endoscopic treatment of pediatric structural incontinence.

Materials and Methods: A total of 33 children and adolescents 5 to 18 years old with severe incontinence due to sphincteric incompetence (exstrophy-epispadias in 13, neuropathic bladder in 16, bilateral ectopic ureters in 4) were enrolled in the study. All but 1 patient wore diapers. Preoperative evaluation consisted of medical history, pad test, urine culture, urinary tract ultrasound and videourodynamics. This evaluation was repeated 6 months and 1 year after treatment and then on a yearly basis. Of the patients 14 had 2 and 1 had 3 treatment sessions to achieve a definitive result. At each evaluation the patient was considered cured-dryness interval of 4 hours confirmed by pad test, significantly improved-minimal incontinence requiring no more than 1 pad a day with less than $10 \mathrm{gm}$. leakage during pad test; and no further treatment required, and treatment failure-no significant improvement. Videourodynamics were mainly useful to study the evolution of the bladder capacity, activity and compliance. Followup after the last injection ranged from 6 to 36 months (mean 18).

Results: The mean injected volume was $3.9 \mathrm{ml}$. (range 1.6 to 12) and the procedure lasted a mean of 30 minutes (10 to 60 ). In the postoperative period 2 patients had temporary dysuria and 10 had a nonfebrile urinary tract infection. At 1 month 24 of the 33 patients (73\%) were dry or improved. Two patients were subsequently excluded from study for noncompliance with followup. At 6 months 17 of 31 patients (55\%), at 1 year 13 of $28(46 \%)$, at 2 years 10 of $23(43 \%)$ and at 3 years 10 of $20(50 \%)$ were dry or improved. Similar success occurred in cases of neuropathic bladder (57\%) and exstrophy-epispadias complex (46\%). Success rate of re-treated patients was $35 \%$. Of 13 patients with a normal initial bladder capacity 3 had a bladder decompensation requiring augmentation after 6 months. Also an increase of at least 50\% in capacity was observed in 12 of 18 patients with an initial small bladder. No side effect related to the substance was observed.

Conclusions: Endoscopic treatment of pediatric structural urinary incontinence with dextranomer implant, a nontoxic, nonimmunogenic, nonmigrant synthétic substance, was effective after 3 years in half of our patients. It may also be beneficial for patients with small bladder functional capacity. As the success rate decreased during the first year of followup, the result observed at 1 year seems to remain stable subsequently.

KEY WoRDS: urinary incontinence; bladder exstrophy; epispadias, endoscopy, dextrans

Sphincteric incompetence in children and adolescents related to neuropathic bladder sphincter dysfunction and the exstrophy-epispadias complex is a major therapeutic challenge for the pediatric urologist. The 2 conditions for successful treatment are achievement of good outlet resistance and a compliant nonoveractive bladder with adequate capacity. Bladder outlet resistance can be improved by major surgical procedures such as bladder neck plasty, slings, urethral lengthening or urinary sphincter. However, these procedures do not reliably achieve a good result for every patient. ${ }^{1}$ In many instances the patients have to perform clean intermittent catheterization to empty the bladder through the urethra or via a continent stoma (Mitrofanoff channel). In case of failure or as a first measure the bladder neck can be closed and a continent catheterizable reservoir created.

* Financial interest and/or other relationship with Q-Med, Inc. and Pharmacia.
Endoscopic injections of bulking agents have been proposed as an alternative to these major reconstructive procedures or as an adjunct if they fail to achieve acceptable social continence. Several agents, such as polytetrafluoroethylene paste, collagen and more recently polydimethylsiloxane have been used to achieve similar respectable results. Considering the simplicity of the procedure and that an average of $50 \%$ of patients are either cured or significantly improved compare favorably with the more aggressive anti-incontinence measures. ${ }^{2}$ Also, when bladder capacity is small due to poor outlet resistance endoscopic injection of bulking agents may be useful to evaluate the ability of the bladder to develop. Recently, a new bulking agent made of dextranomer particles mixed in hyaluronic acid (Deflux, Questor AB, Uppsala, Sweden) has been reported to give excellent long-term results for the endoscopic treatment of vesicoureteral reflux in several hundreds of children. ${ }^{3}$ We designed a prospective non- 
controlled study using dextranomer particles mixed with hyaluronic acid as a bulking agent for the management of severe organic incontinence in children and adolescents. The study protocol was submitted to our regional ethical committee and approved.

\section{PATIENTS AND METHODS}

Patients 5 to 20 years old treated at our institutions for severe incontinence related to sphincter deficiency were evaluated for study inclusion. Exclusion criteria were vesicoureteral reflux, noncontrolled bladder overactivity and poor bladder compliance. An informed consent was obtained from the patient or parents. Initial evaluation included a medical history, estimate of the level of incontinence (major, moderate, minor or absent), pad test, urine culture, renal ultrasound and videourodynamics. The pad test was always applied under the same conditions for each patient. At the beginning of the test the patient received a fluid load adapted to the body weight and $20 \mathrm{gm}$. pad. After an hour of rest the pad was weighed to evaluate the level of incontinence at rest. Then the patient performed a series of exercises and the pad was weighed again to evaluate the level of stress incontinence. The patient was then requested to void or catheterize to evaluate the volume of urine in the bladder at the end of the test. The urine had to be sterile at the time of treatment.

With the patient under general anesthesia patients received an endoscopic injection of dextranomer based implants. All the injections were performed by 2 of the authors (H. L. and G. L.) using a $10 \mathrm{Fr}$ paediatric urethrocystoscope with a $5 \mathrm{Fr}$ working channel and a 30-degree lens. Initially we used a 19 gauge $\times 350 \mathrm{~mm}$. semirigid Deflux needle but as disconnection occurred between the syringe and the needle during injection, it was subsequently replaced with a $5 \mathrm{Fr} 23$ gauge $\times 350 \mathrm{~mm}$. flexible Williams cystoscopic injection needle with a Luer-Lok system (Cook Urological, Spencer, Indiana). Except for the first 9 patients a suprapubic catheter was inserted and left for 5 days before the patient was allowed to catheterize or void again.

Followup at 1 month comprised subjective evaluation of incontinence by the patient and family, pad test, urine culture and renal ultrasound. This evaluation was repeated as well as videourodynamics at 6 months, 1 year and then on a yearly basis. At each evaluation the patient was considered cured-dryness interval at rest or during efforts of 4 hours at least between 2 voids or clean intermittent catheterization confirmed by pad test, significantly improved-minimal incontinence requiring no more than 1 pad a day and with less than $10 \mathrm{gm}$. leakage during the pad test and no further treatment required and treatment failure-no improvement observed. Bladder capacity was classified as initially normal, according to the formula capacity $(\mathrm{cc})=$ [age $($ years $)+2] \times$ 30 ) and stable or reduced after treatment, or initially small and stable or increased after treatment. Cured or significantly improved patients were monitored under regular followup, while those with partial improvement after the initial injection or secondary recurrence of incontinence after an initial positive response were considered for re-treatment. Patients with primary or secondary treatment failure were given alternative options.

Since September 1997, 25 males and 8 females 5 to 18 years old (mean age 10.3) were included in the study. All except 1 wore diapers for major incontinence related to neuropathic bladder (16), bladder exstrophy-epispadias complex (13) or bilateral ectopic ureters (4). Clean intermittent catheterization was used in 18 patients at the time of the initial evaluation. None of the patients with a neuropathic bladder had undergone a previous surgical procedure on the bladder neck to improve continence, whereas 8 patients with exstrophy-epispadias complex had undergone previous
Young-Dees type of bladder neck plasty with bladder augmentation in 3 . The 4 patients with bilateral ectopic ureters had undergone various types of bladder neck plasty. Renal ultrasonography was normal in all patients except 3 girls with a solitary kidney in the context of a Rokitansky Meyer syndrome (1) and renal scars consecutive to previous urinary tract infections (2). The preoperative pad tests as well as the videourodynamics documented major stress incontinence in all patients with a mean urethral pressure profile of $44 \mathrm{~cm}$. water (range 10 to 90). Fifteen patients were on anticholinergic therapy for detrusor instability. With or without anticholinergics, no patient had an uncontrolled overactive bladder at the time of treatment and or vesicoureteral reflux. According to Berger's formula 14 patients had normal and 19 had small bladder capacity for age. These 33 patients received 1 (18), 2 (14) or 3 (1) injections, representing a total of 49 treatment sessions.

Males were injected systematically below the verumontanum in the area of the external sphincter and in most cases along the posterior urethra proximal to the verumontanum. Females were injected all along the urethra. The male patients who had undergone a previous Young-Dees bladder neck plasty were injected along the tube and below the verumontanum. Mean injected volume per session was $3.9 \mathrm{cc}$ (range 1.6 to 12) and mean duration of the procedure was 30 minutes (10 to 60). Results in terms of continence and bladder capacity are reported according to the delay of followup after the last injection and subcategorized according to the initial pathology, previous treatments and sex. Results are reported as trends only, as the series is too heterogeneous and limited to allow statistical analysis.

\section{RESULTS}

Treatment was without complications in most cases, although 2 patients experienced temporary dysuria and 10 had a nonfebrile urinary tract infection, including 1 case of encrusted cystitis that resolved after adequate treatment. In 1 patient postoperative pneumonia developed. None of the 15 patients who were voiding before treatment had to start clean intermittent catheterization after treatment or had significant post-void residual. No adverse effect related to the dextranomer based implants was observed. At 1 month 24 of the 33 patients $(73 \%)$ were dry $(15,46 \%)$ or improved $(9,27 \%)$ and 9 of $33(27 \%)$ were treatment failures. Two patients were subsequently excluded from the study, of whom treatment failed in 1 and the other was dry at 1 month with regular clean intermittent catheterization but refused to catheterize regularly, and both refused any further followup. The remaining 31 patients have been under regular surveillance (fig. 1). For those patients who had several injections followup is considered from the last treatment session. At 6 months 17 of 31 patients $(55 \%)$ were dry $(29 \%)$ or improved $(26 \%)$, at 1 year 13 of $28(46 \%)$ were dry $(25 \%)$ or im-

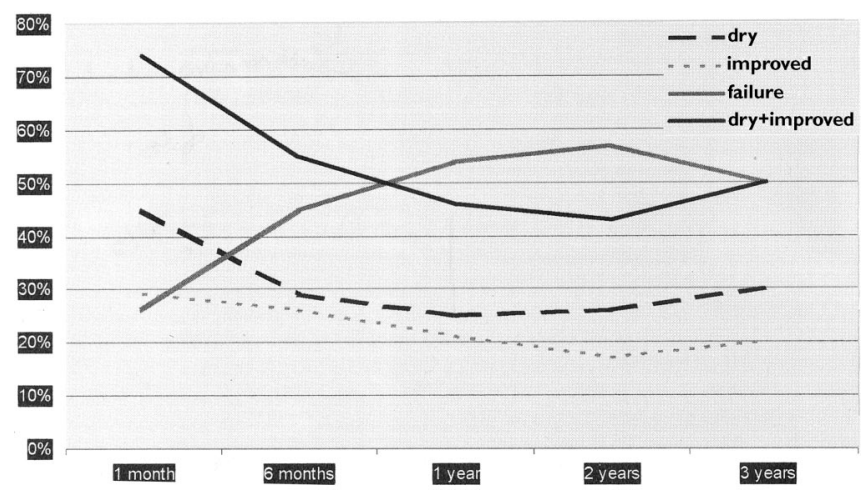

FIG. 1. Sequential results of continence in 3 years of followup 
proved $(21 \%)$, at 2 years 10 of $23(43 \%)$ were dry $(26 \%)$ or improved (17\%) and at 3 years 10 of $20(50 \%)$ were dry $(30 \%)$ or improved (20\%).

Of the 17 patients who were dry or improved at 6 months 4 had recurrent incontinence due to secondary treatment failure (1) and bladder contraction (3). The latter 3 patients underwent bladder augmentation and became dry again with a followup of more than 3 years. None of the patients who were dry or improved after 1 year from the last injection has had recurrent incontinence to date. Of the 14 patients with a neuropathic bladder $8(57 \%)$ are dry (6) or improved (2), and of the 13 patients with bladder exstrophy-epispadias complex $6(46 \%)$ are dry (4) or improved (2), including 3 with classic bladder exstrophy and 3 with posterior epispadias. The 7 patients in this subgroup with treatment failure had classic bladder exstrophy.

As shown in figure 2, previous surgery on the bladder neck was often associated with a poor outcome. Of the 4 patients treated with previous bladder neck surgery for incontinence associated with bilateral ectopic ureters none was dry, 3 were improved and treatment failed in 1 . Gender analysis revealed that 3 of $8(37,5 \%)$ girls and 15 of $23(65 \%)$ boys were dry or improved. Of the 15 patients who received several injections $10(67 \%)$ had immediate failure and $5(33 \%)$ were dry (3) or improved (2). Of the 13 patients with normal initial bladder capacity who were initially dry after a single injection 3 started to leak again after 6 months and videourodynamics revealed reduced bladder capacity and compliance. In 1 of these patients vesicoureteral reflux developed to the solitary kidney (fig. 3). These 3 patients underwent detrusorotomy and were dry after more than 2 years of followup.

No significant change was observed in 6 of the 18 patients with a small bladder capacity and the volume increased by at least $50 \%$ over initial capacity in 12 . Evolution of the bladder capacity according to the expected capacity for age in these 12 patients is shown in figure 4 . The effect on bladder capacity was independent of the effect on continence among these 12 patients, and 1 was dry, 5 were improved, and treatment failed in 6 . Of the 14 patients in whom endoscopic treatment failed to improve their continence, 2 had an artificial urinary sphincter implanted and are now dry, 2 underwent a sling procedure with augmentation and are improved and 3 underwent bladder neck plasty, following which 1 is dry and 2 are improved after short followup. The other 7 patients await further treatment.

Urodynamic studies proved particularly useful to follow the reactions of the bladder after treatment but no correlation could be made between the urethral pressure profile, which most of the time did not vary significantly, and outcome. The 3 patients with unstable bladder contractions after treatment had high initial urethral pressure profiles (mean $70 \mathrm{~cm}$. water). In 3 male patients urethral pressure profile

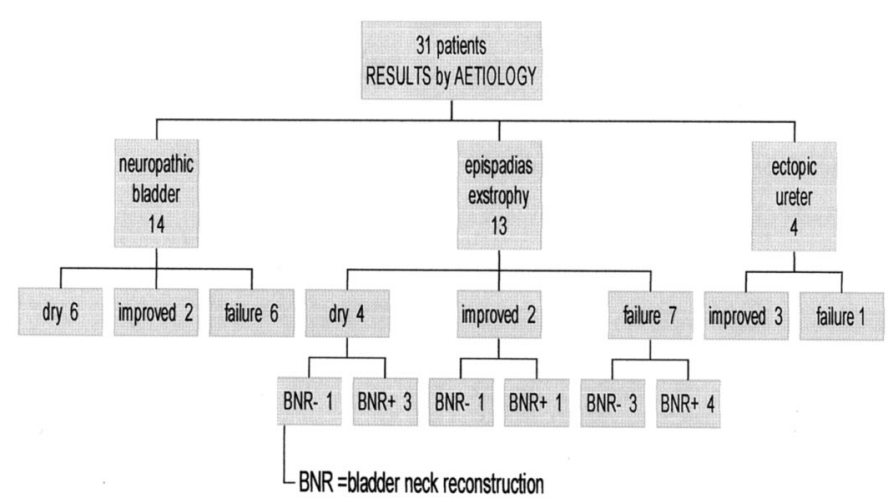

FIG. 2. Results according to initial pathology. BNR, bladder neck reconstruction. increased after puberty (mean $120 \mathrm{~cm}$. water), and all of them were dry with lower urethral pressure profiles before puberty. A contracted bladder developed in 1 of these patients. Such high urethral pressures have not been recorded after puberty in patients who have remained incontinent after endoscopic treatment.

\section{DISCUSSION}

The main purpose of our study was to evaluate prospectively dextranomer particles as a bulking agent for endoscopic treatment of sphincter incompetence after a reasonable period of followup. Endoscopic treatment of incontinence is not a new concept in adults or children. In 1985 Vorstman et al reported on 11 children treated for urinary incontinence with 1 or more endoscopic injections of polytetrafluoroethylene into the external sphincter region and claimed cure or improvement in $8 .{ }^{4}$ Since then, this technique has been used by numerous authors generally in limited numbers of children. Numerous substances have been proposed as bulking agents. ${ }^{1}$ Polytetrafluoroethylene paste was most frequently reported initially but has been abandoned in children as well as in adults. Glutaraldehyde cross-linked collagen was first reported in children by Wan et $\mathrm{al}^{5}$ and more recently use of polydimethylsiloxane particles was reported by Duffy and Ransley. ${ }^{6}$ With glutaraldehyde cross-linked collagen, the reported success rates vary from $53 \%$ to $76 \% .^{7-12}$

The largest series with the best results and the longest followup was reported by Bomalaski et al. ${ }^{13}$ They treated 40 children, including 25 with neuropathic bladder and 12 with the exstrophy-epispadias complex, with 70 glutaraldehyde cross-linked collagen injections and reported a $76 \%$ success rate (only $22 \%$ cured and $54 \%$ improved). They also reevaluated a subgroup of 7 patients after a mean followup of 4.5 years and confirmed that the cure or improvement rate remained stable with time.

Experience with polymethylsiloxane particles is more limited; Duffy and Ransley treated 12 male patients with epispadias, none of whom had undergone previous surgery on the bladder neck, with 24 endoscopic injections and obtained a complete cure in 3 and improvement in 6 (success rate 75\%). ${ }^{6}$ They also observed an increase in bladder capacity in most of their patients including 1 of the 3 who remained incontinent. Guys et al reported on a series of 33 patients with neuropathic bladder-sphincter dysfunction, including 15 who had undergone a surgical procedure on the bladder neck. ${ }^{14}$ After 1 to 3 injections (total 46 injections) and mean followup of 16 months (range 6 to 41) complete cure was achieved in 11 cases $(33 \%)$ and improvement in $8(24 \%)$.

Although our series is limited and statistical analysis was not performed, our results with a cure rate of $30 \%$ and improvement rate of $20 \%$ at 3 years of followup match the results of the other series. Also most authors report global results, including patients with short-term and long-term followup, which optimizes the overall success rate as during the first year after treatment incontinence may recurr in a significant number. There is an obvious need to standardize the evaluation of the incontinence as the interpretation of results varies considerably.

Our results were similar for neuropathic bladder-sphincter dysfunction and the exstrophy-epispadias complex. In contrast to other series, in which poor results are achieved in patients with neuropathic bladder-sphincter dysfunction, none of our patients had undergone previous surgery on the bladder neck. In general, we achieved poorer results in patients after failed bladder neck or Young-Dees procedures and, thus endoscopic injection for incontinence probably should be considered as primary therapy rather than a salvage procedure. Our results were also better in males than in females but the discrepancy in the numbers in each category is such that the 2 groups are not comparable. We agree with 

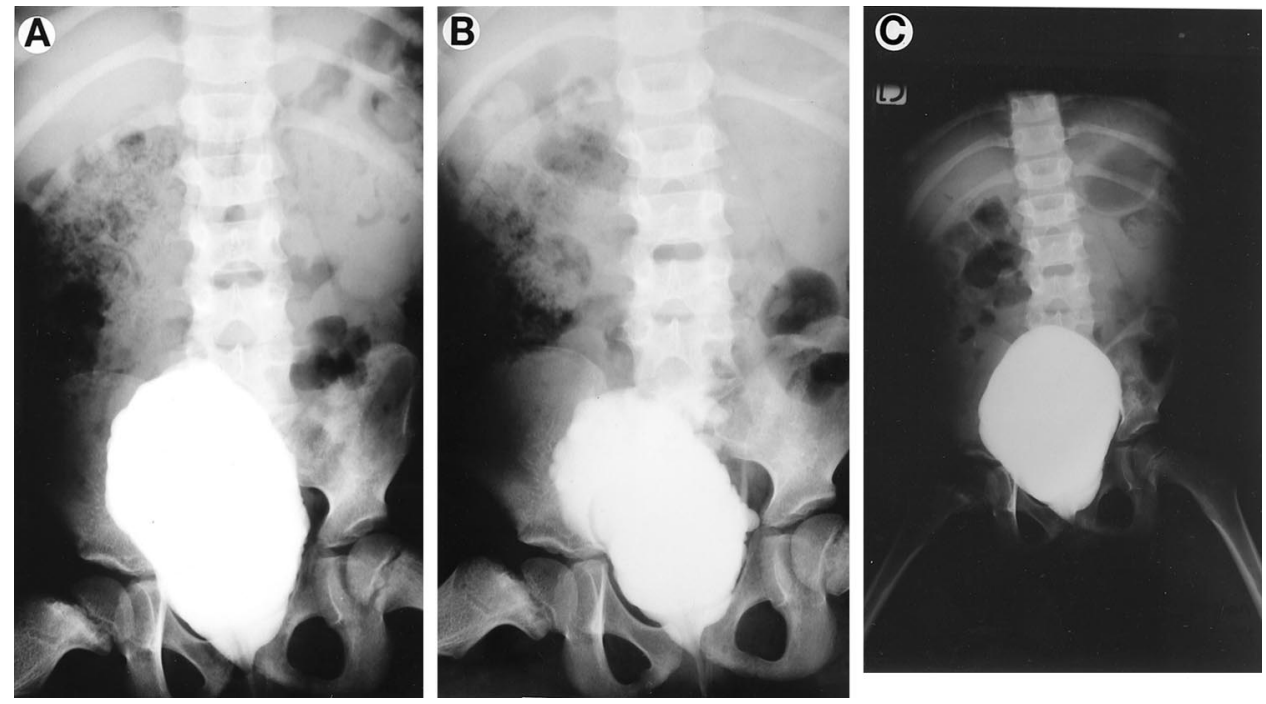

FiG. 3. Bladder deterioration 6 months after single successful injection in 7-year-old girl with neuropathic bladder and partial sacral agenesis with Rokitansky-Meyer syndrome. $A$, pretreatment cystogram. $B$, recurrence of incontinence associated with reduction in bladder capacity and appearance of vesicoureteral reflux to solitary kidney. $C$, dry again 3 years after detrusorotomy.

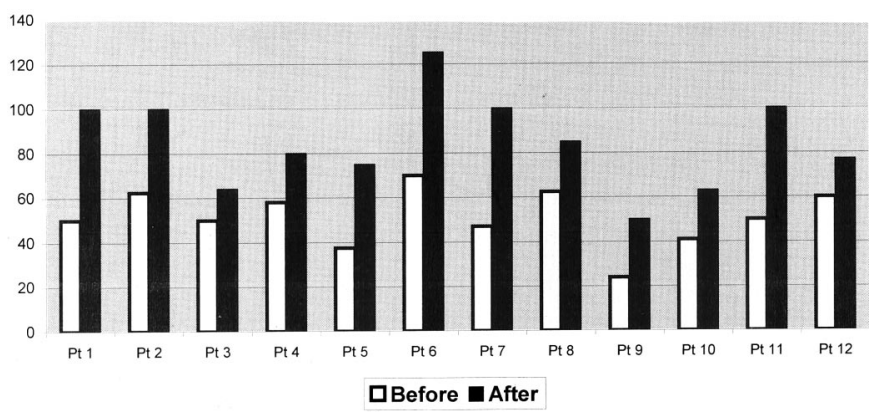

Fig. 4. Increase in bladder capacity after dextranomer implant in 12 patients (\% of normal capacity for age).

Bomalaski et al ${ }^{13}$ that after 1 year the results achieved are stable, although longer followup of a larger number of patients is necessary to confirm this fact. Like Chernoff et al, ${ }^{11}$ we observed bladder deterioration in 3 of our patients to date. Thus in a patient with an initial success for a few months, recurrence of incontinence may indicate detrusor deterioration rather than bulking agent reabsorption. Such patients should undergo videourodynamic evaluation before making any conclusions. Urodynamics are clearly essential to follow the natural history of the bladder but evolution of outlet resistance is much more controversial, and there is a need for standardization of the protocols.

We did not find a significant increase in pressure profile after treatment in any of our patients, although those who have high initial pressures may represent a subgroup at risk for further bladder deterioration. Perez et al could not correlate Valsalva leak pressures with their continence results. ${ }^{9}$ However, Chernoff et al did find a correlation between success and an increase in Valsalva leak point pressure from 20 to $25 \mathrm{~cm}$. water to greater than $60 \mathrm{~cm}$. water, ${ }^{11}$ and Bomalaski et al reported similar observations. ${ }^{13}$ We perform urodynamics using a transurethral and not a suprapubic catheter, and we do not consider leak point pressure a reliable parameter in such conditions. Similar to Duffy and Ransley, ${ }^{6}$ we observed a significant increase in bladder capacity in patients with an initially small bladder. When compared to the normal bladder capacity for age during followup, it was obvious that for most patients improvement was secondary to growth. However, if patients did not achieve normal capacity it must be noted that disabled patients often have a small bladder for age.
Patient selection is more important than the nature of the bulking agent, since all agents seem to provide similar success rates. However, dextranomer particles (80 to $250 \mu \mathrm{m}$. microspheres) in a gel of hyaluronic acid may have some advantages. As a synthetic product, dextranomer and hyaluronic acid are biodegradable, and are neither allergenic nor immunogenic. Experimental studies have failed to demonstrate distant migration. ${ }^{15,16}$ Following injection dextranomer facilitates ingrowth of fibroblasts and collagen between the microspheres as hyaluronic acid is degraded. Thus, the bolus is consolidated with endogenous tissue, stabilizing its volume for a sustained, long-term response. Dextranomer has been used for the treatment of vesicoureteral reflux in several hundred of children with long-term results similar to those of other bulking agents. ${ }^{3-17}$ The product is particularly easy to inject even through a thin needle without the need for a gun. However for endourethral injections the depth and pressure of injection are greater than those for the treatment of vesicoureteral reflux. Using the initial thin needle we encountered the problem of a disconnection of the syringe from the needle. Use of the Williams cystoscopic injection needle improved our technique of injection and our results.

\section{CONCLUSIONS}

Although the number of patients included in the study is too small to allow statistical analysis and, thus, only the trends are reported, dextranomer implants seem to give similar results to other bulking agents for the treatment of severe organic incontinence in children. After 1 year results remain stable for more than 3 years. Recurrence of incontinence after initial success may be evidence of bladder degradation. Endoscopic injection may increase bladder capacity independently of the results of continence. Even if the complete cure rate is low, endoscopic treatment should be considered as a first option in this group of patients, since a definitive cure with major reconstructive surgical procedures cannot be constantly achieved. Our review of other series obviates the need to standardize the way endoscopic treatment is evaluated.

Dr David Bloom revised the manuscript.

\section{REFERENCES}

1. Casale, A. J.: Assessment and current management of bladder outlet incontinence in children. In: Atlas of the Urologic 
Clinics of North America. Edited by R. C. Rink. Philadelphia: W. B. Saunders Co., chapt. 9.1, p. 119, 2001

2. Bomalaski, M. D., Koo, H. P. and Bloom, D. A.: Injectable bulking agents in the treatment of urinary incontinence. In: Pediatric Urology. Edited by J. P. Gearhart, R. C. Rink and P. D. E. Mouriquand. Philadelphia: W. B. Saunders Co., chapt. 66 , p. 1008,2001

3. Läckgren, G., Wåhlin, N., Sköldenberg, E. and Stenberg, A.: Long-term followup of children treated with dextranomer copolymer for vesicouretral reflux. J Urol, 166: 1887, 2001

4. Vorstman, B., Lockhart, J. L., Kaufman, M. and Politano, V. A.: Polytetrafluoroethylene injection for urinary incontinence in children. J Urol, 133: 248, 1985

5. Wan, J., McGuire, E. J., Bloom, D. A. and Ritchey, M. L.: The treatment of urinary incontinence in children using glutaraldehyde cross-linked collagen. J Urol, 148: 127, 1992

6. Duffy, P. G. and Ransley, P. G.: Endoscopic treatment of urinary incontinence in children with primary epispadias. Br J Urol, 81: 309, 1998

7. Ben-Chaim, J., Jeffs, R. D., Peppas, D. S. and Gearhart, J. P.: Submucosal bladder neck injections of glutaraldehyde crosslinked collagen for the treatment of urinary incontinence in patients with the exstrophy/epispadias complex. J Urol, 154: 862,1995

8. Leonard, M. P., Decter, L. W., Mix, L. W., Johnson, H. W. and Coleman, G. U.: Treatment of urinary incontinence in children by endoscopically directed bladder neck injection of collagen. J Urol, 156: 637, 1996

9. Perez, L. M., Smith, E. A., Parrott, T. S., Broecker, B. H., Massad, C. A. and Woodard, J. R.: Submucosal bladder neck injection of bovine dermal collagen for stress urinary incontinence in the pediatric population. J Urol, 156: 633, 1996

10. Silveri, M., Capitanucci, M. L., Mosiello, G., Broggi, G. and De Gennaro, M.: Endoscopic treatment for urinary incontinence in children with a congenital neuropathic bladder. Br J Urol, 82: 694, 1998

11. Chernoff, A., Horowitz, M., Combs, A., Libretti, D., Nitti, V. and Glassberg, K.: Periuretral collagen injection for the treatment of urinary incontinence in children. J Urol, 157: 2303, 1997

12. Capozza, N., Caione, P., De Gennaro, M., Nappo, S. and Patricolo, M.: Endoscopic treatment of vesico-ureteric reflux and urinary incontinence: technical problems in the paediatric patient. Br J Urol, 75: 538, 1995

13. Bomalaski, M. D., Bloom, D. A., McGuire, E. J. and Panzl, A.: Glutaraldehyde cross-linked collagen in the treatment of urinary incontinence in children. J Urol, 155: 699, 1996

14. Guys, J. M., Simeoni-Alias, J., Fakhro, A. and Delarue, A.: Use of polydimethylsiloxane for endoscopic treatment of neurogenic urinary incontinence in children. J Urol, 162: 2133, 1999

15. Stenberg, A., Larsson, G., Johnson, P., Heimer, G. and Ulmsten, U.: DiHA dextran copolymer, a new biocompatible material for endoscopic treatment of stress incontinent women. Acta Obstet Gynecol Scand, 78: 436, 1999

16. Stenberg, A. M., Sundin, A., Larsson, B. S. et al: Lack of distant migration after injection of a 125 iodine labeled dextranomer based implant into the rabbit bladder. J Urol, 158: 1937, 1997

17. Capozza, N. and Caione, P.: Dextranomer copolymer implantation for vesico-ureteral reflux: a randomized comparison with antibiotic prophylaxis. J Pediatr, 140: 230, 2002

\section{DISCUSSION}

Dr. Miguel Podesta. Have you analyzed your results according to the underlying pathology of your study population?

Dr. Henri Lottmann. Yes. The results are slightly better in the neuropathic bladder than in the exstrophy group. There were about $60 \%$ satisfactory results in the neurogenic bladder group. This is why it is important that these are primary cases with no previous surgery on the bladder neck. In my exstrophy-epispadias group the results were also better in the primary epispadias cases. 